\title{
Qualitative Assessment of a Clutch-Actuated Ankle Exoskeleton
}

\author{
Miha Dežman ${ }^{1,2}$, Jan Babič ${ }^{1}$ and Andrej Gams ${ }^{1,2}$ \\ 1 Dept. of Automation, Biocybernetics and Robotics, \\ Jožef Stefan Institute, \\ Ljubljana, Slovenia \\ 2 Jožef Stefan International Postgraduate School, \\ Ljubljana, Slovenia, \\ miha.dezman@ijs.si
}

\begin{abstract}
Among the growing number of different exoskeletons, passive and quasi-passive solutions hold the upper-hand compared to powered solutions in price, accessibility, complexity, weight and user acceptance. This paper evaluates a modification of an originally passive ankle exoskeleton with an active clutch, making it quasi-passive. We developed an electric quasi-passive clutch to improve the performance of the original exoskeleton design, with the aim of mitigating the problems on the clutch engagement timing and user physiological variability. In order to evaluate the exoskeleton and the clutch operation, we performed a study where 7 users wore the exoskeleton and performed trial walks. Qualitative user feedback that focused on the device comfort, users perception of exoskeletons effect and smoothness of the clutch operation was collected, along with quantitative data on clutch operation during walking on a flat surface. Results show improved and more reliable exoskeleton clutch operation which was also expressed in qualitative user feedback.
\end{abstract}

Keywords: Ankle Exoskeleton, Electric clutch, Quasi-passive, Wearable exoskeleton

\section{Introduction}

Among the different types of robotic devices intended to help humans, exoskeletons might be some of the most promising. Due to recent advancements in technology, their use in everyday lives is closer than ever [1]. Recently, several devices have been developed to the point of entering the market for everyday end-users. The web-site Exoskeleton Report [2] shows a comprehensive list of current actual exoskeletons. These differ in the target application ranging from measuring devices to assistance in industrial and home environments, in order to relieve the stress of users/workers, and to medical applications. The last one includes increasing mobility and autonomy of people with disabilities and rehabilitation tasks for both upper and lower extremities. Some advantages for the use of exoskeleton devices in rehabilitation are presented in [3]. One of intended uses of 


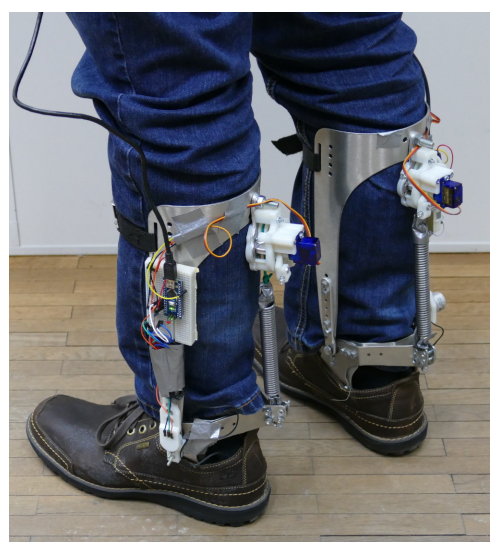

Fig. 1: User wearing the exoskeleton.

exoskeletons is the reduction of metabolic cost of human activities. However, powered exoskeletons can, due to their weight and kinematic constraints, often increase metabolic cost of the user. Reduction in metabolic cost can be achieved in different manners. One of them is in minimal weight of the mechanism and active assistance, which has led to the development of soft exo-suits [4]. Besides their low weight these also do not impose additional kinematic constraints on the user. Another way to reduce the metabolic cost is the manner of actuation which has been shown to have a direct influence on the metabolic cost of squatting [5]. Other tasks have also been studied, e.g., a review on different control methods for robotic ankle systems is available in [6], while an experimental comparison between different torque methods for ankle exoskeletons is presented in [7].

Besides the different actuated exoskeletons, passive devices also exist. These are not actuated in normal sense, but non-the-less still reduce the effort needed to perform a human activity. An example are the passive tool-holding exoskeletons. The Ekso Works exoskeleton [8], developed by Exo Bionics, balances a workers tool that is fixed on a pre-tensioned spring-arm mechanism that transfers the tool weight to the ground. This way the work load of the tool on the user is reduced. Another similar exoskeleton was developed by Lockheed Martin and is called Fortis [9]. Other passive exoskeleton devices can use passive elements to store energy during one part of the task in order to release it in another part.

Passive storage of energy is also observed in humans. Tendons, acting as springs, can passively store energy in periodic human activities, like walking and running. Animals and humans evolved three different musculo-skeletal systems that can store energy [10]. The first is bouncing along on springs while running (pogo-stick principle) by using monoarticular (across one joint) and multiarticular muscles and tendons. Another way to store energy is to use return springs to change the direction of a moving link. The third way is the use compliant foot-pads, which reduce impact forces to the ground. These methods have been applied in the design of different exoskeletons and robots.

For example Chin et al. [11] presented a pneumatic foot pad exoskeleton that is able to harvest the power of a foot drop. Dollar et al. [12] developed a quasi- 
passive exoskeleton to assist in running. Quasi-passive exoskeletons use power to control an exoskeleton (for example a brake or clutch), but do not input power into joints. Research group Grant et al. [13] developed a passive elastic knee exoskeleton for running. Furthermore, Dijk et al. [14] developed the XPED, a passive lower limb exoskeleton with artificial multi-articular tendons.

The ankle joint has received a lot of attention in both active and passive exoskeletons. Recently, Collins et al. [15] developed a passive ankle exoskeleton that uses a spring connected in parallel with the human calf and a mechanical clutch. The spring, which is operated by a passive mechanical clutch, is able to store and return energy to the ankle at correct times and thus reduce the effort of human walking for an average of $7 \%$. Since the original publication, they proposed several modifications, including an even more light-weight electroadhesive clutch and spring [16]. Besides the reduced weight, the advantage of using an active electroadhesive clutch is in the increased reliability of locking.

In our previous paper [17] we reported on an evaluation of a self-built passive exoskeleton based on the original presented by Collins et al. [15]. One of the main finding from our paper [17] is that the design of the clutch relies on the footground impact to engage the pawl and thus lock the clutch. However, the pawl engaging and disengaging has not been reliable and was reported by many test subjects as one of the main issues with the device. In this paper we thus report on a modification of the original mechanism by Collins et al. [15] with an actuated clutch. The modified quasi-passive ankle exoskeleton was tested by 7 subject while walking on a flat terrain. We gathered qualitative user feedback through a questionnaire in order to asses their comfort with the device and its operation.

This paper is organized as follows. In the next Section we present the modification of the ankle exoskeleton to include an actuated clutch. In Section 3 we provide the experimental protocol, followed by the evaluation of the results in Section 4. A conclusion and the prospects of future improvements are given in Section 5 .

\section{Mechanics and Operation}

The original passive ankle exoskeleton we developed relied heavily on pre-tuned clutch activation timing. However, this timing differs from person to person and even for a person walking using different style, speed and stride length. The on-off operation was dependent on the position of the engagement pins and the length of the rope connecting the clutch with the spring.

The clutch operation was thus highly unreliable and hard to tune which also prevented the device to operate at full potential. By using an electrical clutch these problems would all be solved. In this work, a small servo motor was integrated into the clutch to make its operation more reliable. This increased the overall weight of the device by only $280 \mathrm{~g}$, and did thus not impose considerable additional effort on the user.

Since the pawl only needs a small push to (dis)engage, the actuator can be small and of low power with a very low energy consumption. The ratchet and 
pawl clutch mechanism can be seen in Fig. 2. The added active clutch makes the exoskeleton quasi-passive. The full exoskeleton device can be seen in Fig. 3. Additionally, it now also features a foot switch placed at the heel. The micro servo motor is attached on the clutch above the pawl. A small spring is added between the servo motor arm and the pawl to act as a shock absorber and filter out the shock loads resulting from the pawl engagement and disengagement. This protects the plastic gears inside the micro servo motor.

The exoskeleton permits only one degree of motion (ankle plantar-flexion and dorsiflexion). A magnetic rotor encoder sensor is used to measure the angle at the exoskeleton joint. A linear spring is placed between the foot frame and the upper calf frame where it is connected to the clutch with a rope. If the clutch is closed, the ankle joint movement results in the extension of the linear spring. And if the clutch is open, the linear spring does not affect the exoskeleton movement. A smaller return spring connects the pulley system to

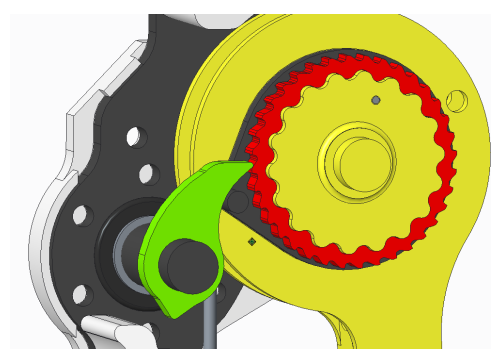

Fig. 2: The ratchet (red) and pawl (green). the frame of the clutch which prevents the slacking of the rope that is used to connect the linear spring to the clutch system.

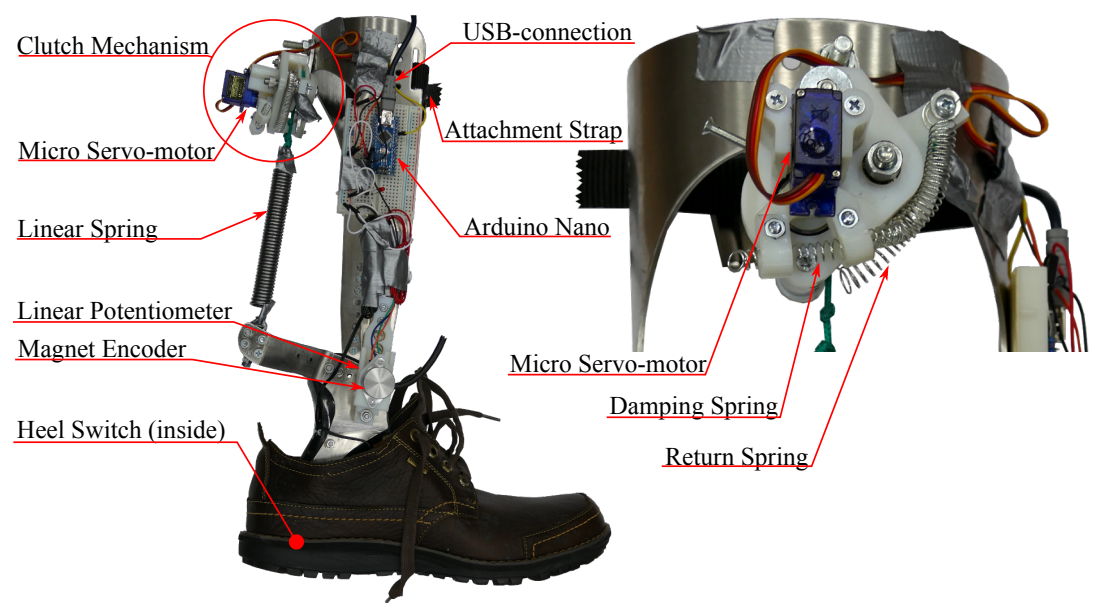

Fig. 3: Exoskeleton prototype and the quasi-passive clutch mechanism.

Each exoskeleton hosts an Arduino Nano that serves as the system controller. The Arduino is used to collect data from sensors and to control the micro servo motor. In this study, the USB cable connection from the Arduino to a laptop served as both the power and communication port. The laptop computer was placed in a backpack so that the subjects could carry the system around while 
the data was being logged. The algorithm running on the Arduino is constructed to run endlessly and send the data to the laptop upon a request. The Arduino internal control algorithm was running with a frequency of $1000 \mathrm{~Hz}$ while the laptop sampled the data at $100 \mathrm{~Hz}$.

During operation, the Arduino detects the heel strike and orders the servo motor to close the clutch. Next, when the heel-switch is released, the servo is given a command to open the clutch. However, since the pawl is under load, the servo is unable to open it due to its low power. Thus, the clutch stays closed until the linear spring gets unloaded which is exactly the desired behavior of the spring. The extension spring gets loaded and stores energy that is returned at the push-off phase. At this point the spring is completely unloaded which means that the motor is able to open the clutch. The exoskeleton operation principle is shown in Fig. 4. The Figure also presents the heel-switch activation and the corresponding exoskeleton angle. During the experiments we collected the data, i. e., the ankle angle and the heel-switch value (open, closed) at both ankles.

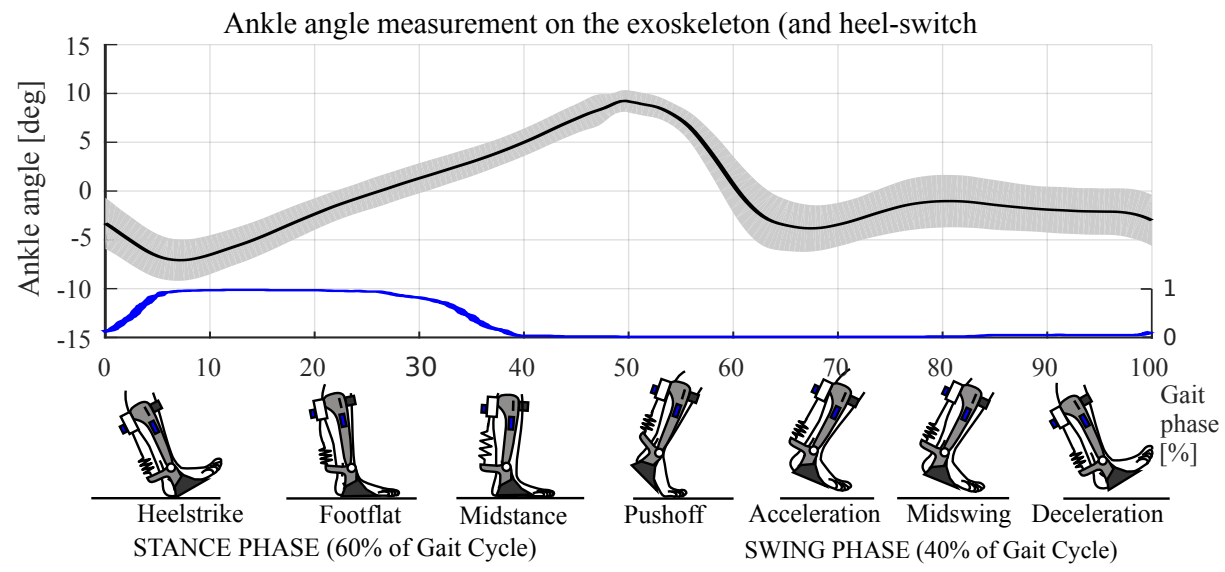

Fig. 4: Average angle (in black) and its standard deviation (in gray) measured at the exoskeleton joint in relation to the gait phase. Heel-switch activation probability is shown in blue and is calculated for a 100 steps.

\section{$3 \quad$ Experimental Design and Protocol}

In order to evaluate the exoskeleton, we performed a pilot study comprised of three 4-minute walks under different conditions. The trial's prime focus was on the operation of the quasi-passive clutch. Thus, from the users point of view, no extensive pre-trial user preparation was needed. The users donned the exoskeleton and started walking. Since the separate walks were relatively short, the time needed for the examiner to prepare the user for the next walk was also enough for the user to rest. The three walking conditions, tested in a cross-over design, were as follows: one represented the reference walk with the exoskeleton and no spring. 
Another walk used the $100 \%$ rate clutch operation and a $5 \mathrm{~N} / \mathrm{mm}$ spring. In the last case the exoskeleton used the same spring, but with only an $80 \%$ chance of clutch closure, meaning that in 100 steps, the clutch would randomly not close 20 times. The opening and closing of the clutch was specified in the algorithm running on the micro controller. Thus we simulated a miss-performing clutch as it was observed in the first prototype.

The walking was done in the lab hallway in path shaped like a square of size $15 \times 10 \mathrm{~m}$. This means that the users also had to make 4 sharp corner turns while walking. All the required data was collected by the portable computer placed inside the users backpack.

The user were asked to fill out a questionnaire at the end of each trial. The questionnaire was aimed to receive feedback on the exoskeleton ergonomics and comfort, smoothness of the clutch operation and the user feeling of the exoskeleton effects. Each question had four possible answers, ranging from 1 to 4 , where one was specified as the worst and four as the best answer. Also other general operation or device remarks were collected in the last section of the questionnaire. Seven healthy young males were asked to participate in the study. Their baselines characteristics are shown in Table 1.

Table 1: Baseline participant characteristics

\begin{tabular}{|c|c|c|}
\hline & Mean & STD \\
\hline \hline Age (years) & 27.1 & 3.48 \\
\hline Weight $(\mathrm{kg})$ & 75.9 & 9.48 \\
\hline Height $(\mathrm{cm})$ & 176.9 & 5.17 \\
\hline Shoe size $(/)$ & 42.5 & 0.99 \\
\hline
\end{tabular}

\section{Evaluation}

The gathered questionnaires results are of highly subjective nature and should be analyzed with great caution. Nevertheless, they hold valuable information about the exoskeleton usage from the users perspective. One can also deduct several ways of improvement and research direction from this data.

It was observed that the differences in user physiology (foot size and shape) and their walking pattern, have quite a big impact on the operation of the exoskeleton. The heel-switch activation speed and time were dependent on the users heel strike strength, meaning that a secondary condition would have to be taken into consideration in order to achieve $100 \%$ correct clutch activation irrelevant of the user. One could, for example, use the joint angle data and an observer to predict the clutch activation rate, i. e., using adaptive frequency oscillators, similarly as was reported for torque activation in [5]. By analyzing the general remarks and talking to the users, several aspects of the exoskeleton came to the forefront. A few user felt that the clutch in the $80 \%$ run did indeed malfunction. Unfortunately, they were unable to reflect their observations in the questionnaire. The ergonomics and comfort were in the acceptable ranges. 
The comfort level, however, is still not high enough for a prolonged usage. We believe that the slight discomfort also had a negative effect on the users feel of effort reduction. Some users also pointed at the introduced movement restriction resulting from the exoskeletons only degree of movement freedom. Due to this, it is difficult to make sharp turns. In order to perform a $90^{\circ}$ turn, the user needs to walk in a radius with a non-negligible distance. Therefore a frame with a higher number of movement degrees would be desirable in order to improve the exoskeleton.

The averages values for the questionnaire answers are gathered in Table 2 .

Table 2: The average value (and SD) of the questionnaire (the values are between 1 and 4 , where 4 means best).

\begin{tabular}{l||c|c|c|} 
& $\begin{array}{c}1 . \text { walk } \\
\text { average }(\mathrm{SD}) \\
\text { (no spring) }\end{array}$ & $\begin{array}{c}2 . \text { walk } \\
\text { average (SD) } \\
\text { (100\% rate) }\end{array}$ & $\begin{array}{c}3 . \text { walk } \\
\text { average (SD) } \\
\text { (80\% rate) }\end{array}$ \\
\hline \hline Comfort, ergonomics & $2.6 / 4(0.6)$ & $2.7 / 4(0.6)$ & $2.6 / 4(0.5)$ \\
Walking effort reduction & $2.0 / 4(0)$ & $2.7 / 4(0.6)$ & $2.6 / 4(0.7)$ \\
Smooth operation & $3.8 / 4(0.4)$ & $3.1 / 4(0.5)$ & $3.3 / 4(0.6)$
\end{tabular}

The users described the comfort level between a 2 and 3 (out of 4 ). This points out that the exoskeleton is of reasonable comfort, but that there is still room for improvement. We can observe quite a big standard deviation in all cases, meaning that there is a lot of variance between users. Generally, the users do claim to feel some walking effort decrease. However, an intensive study going beyond this pilot evaluation would be needed to verify their claims. As for the clutch operation, the users confirmed that the clutch operation has very little malfunctions.

\section{Conclusion and Future Work}

The quasi-passive exoskeleton upgrade has resulted in a more reliable performance of the device with almost no clutch malfunctions during walking. However, our self-build prototype holds some room for improvement. One issue is the total weight of the exoskeleton, resulting mainly from the stainless steel frame. With the added clutch actuation it now weighs $1090 \mathrm{~g}$ (shoes excluded). In order to reduce the exoskeletons effect on the user, its weight should be minimized. A challenging problem are also the introduced kinematic obstructions the exoskeleton imposes on the user, since it allows only one movement degree of freedom. It is however not clear, to which extent this problem effects the walking effort. All this combined with the ergonomics and comfort, which are still not at the desired levels, also masks the users feeling of walking effort reduction.

The development of the original exoskeleton presented by Collins et al. [15] and the self-built version evaluated in [17] opened several additional research opportunities. It is still an open research question what is the optimal spring stiffness for each user. At this stage, a third prototype is in planning which will foremost be more comfortable and lighter. 


\section{References}

1. A. M. Dollar and H. Herr, "Lower extremity exoskeletons and active orthoses: challenges and state-of-the-art," IEEE Transactions on Robotics, vol. 24, no. 1, pp. 144-158, 2008.

2. "Exoskeleton Report: Exoskeleton Catalog." http://exoskeletonreport.com/ product-category/exoskeleton-catalog/, 2016. Accessed: 2016-01-23.

3. D. P. Ferris, G. S. Sawicki, and A. Domingo, "Powered lower limb orthoses for gait rehabilitation," Topics in spinal cord injury rehabilitation, vol. 11, no. 2, p. 34, 2005.

4. A. T. Asbeck, S. M. M. D. Rossi, I. Galiana, Y. Ding, and C. J. Walsh, "Stronger, smarter, softer: Next-generation wearable robots," IEEE Robotics Automation Magazine, vol. 21, pp. 22-33, Dec 2014.

5. A. Gams, T. Petrič, T. Debevec, and J. Babič, "Effects of robotic knee exoskeleton on human energy expenditure," IEEE Transactions on Biomedical Engineering, vol. 60 , no. 6 , pp. 1636-1644, 2013.

6. R. Jimenez-Fabian and O. Verlinden, "Review of control algorithms for robotic ankle systems in lower-limb orthoses, prostheses, and exoskeletons," Medical engineering 85 physics, vol. 34, no. 4, pp. 397-408, 2012.

7. J. Zhang, C. C. Cheah, and S. H. Collins, "Experimental comparison of torque control methods on an ankle exoskeleton during human walking," in IEEE International Conference on Robotics and Automation (ICRA), pp. 5584-5589, IEEE, 2015.

8. "Ekso Bionics: EksoWorks Exoskeleton." http://eksobionics.com/eksoworks/, 2016. Accessed: 2016-01-23.

9. "Lockheed Martin: Fortis Exoskeleton." http://www.lockheedmartin.com/us/ products/exoskeleton/industrial.html, 2016. Accessed: 2016-01-23.

10. R. Alexander, "Three uses for springs in legged locomotion," International Journal of Robotics Research, vol. 9, no. 2, pp. 53-61, 1990.

11. R. Chin, E. T. Hsiao-Wecksler, E. Loth, G. Kogler, S. D. Manwaring, S. N. Tyson, K. A. Shorter, and J. N. Gilmer, "A pneumatic power harvesting ankle-foot orthosis to prevent foot-drop," Journal of neuroengineering and rehabilitation, vol. 6, no. 1, p. 19, 2009.

12. A. M. Dollar and H. Herr, "Design of a quasi-passive knee exoskeleton to assist running," in IEEE/RSJ International Conference on Intelligent Robots and Systems 2008, pp. 747-754, IEEE, 2008.

13. G. Elliott, G. S. Sawicki, A. Marecki, and H. Herr, "The biomechanics and energetics of human running using an elastic knee exoskeleton," in Rehabilitation Robotics (ICORR), 2013 IEEE International Conference on, pp. 1-6, IEEE, 2013.

14. W. van Dijk, H. van der Kooij, and E. Hekman, "A passive exoskeleton with artificial tendons: Design and experimental evaluation," in IEEE International Conference on Rehabilitation Robotics (ICORR) 2011, pp. 1-6, IEEE, 2011.

15. S. H. Collins, M. B. Wiggin, and G. S. Sawicki, "Reducing the energy cost of human walking using an unpowered exoskeleton," Nature, vol. 522, no. 7555, pp. 212-215, 2015.

16. S. Diller, C. Majidi, and S. H. Collins, "A lightweight, low-power electroadhesive clutch and spring for exoskeleton actuation," in 2016 IEEE International Conference on Robotics and Automation (ICRA),, pp. 682-689, IEEE, 2016.

17. M. Dežman, T. Debevec, J. Babič, and A. Gams, "Effects of passive ankle exoskeleton on human energy expenditure: Pilot evaluation," in International Conference on Robotics in Alpe-Adria Danube Region, pp. 491-498, Springer, 2016. 